hormone-refractory prostate cancer, Fromont et al. suggest that some of the differentially expressed genes studied here might represent useful targets for therapy.

Original article Fromont G et al. (2005) Differential expression of 37 selected genes in hormone-refractory prostate cancer using quantitative taqman real-time RT-PCR. Int J Cancer 114: 174-181

\section{Mechanism of drug resistance in non-small-cell lung cancer}

A recent report by Kobayashi et al. sheds light on the mechanism of drug resistance in nonsmall-cell lung cancer patients who are initially responsive to anilinoquinazoline epidermal growth factor receptor (EGFR) inhibitors.

These drugs - namely gefitinib and erlotinibare used for second-line or third-line therapy in patients with metastatic disease, and work by targeting the EGFR pathway. The majority of highly responsive tumors bear activating mutations of the EGFR gene. All patients appear to relapse eventually, however, and Kobayashi and colleagues hypothesized that this might be due to further somatic EGFR mutations.

They describe the case of a 71-year-old patient with advanced non-small-cell lung cancer who had a clinical and radiographic response to gefitinib after failing chemotherapy. After two years of complete remission, however, the patient relapsed and has since been treated with salvage therapies for advanced lung cancer.

Using genomic DNA extracted from a tumor specimen from the patient, the tyrosine kinase domain of the EGFR gene was sequenced and the results compared with those from the first biopsy. This revealed a second EGFR mutation-a novel $\mathrm{C} \rightarrow \mathrm{T}$ transition in exon 20-which resulted in a threonine-to-methionine substitution at position 790 of the protein. In vitro analysis demonstrated that this mutation, named T790M, conferred gefitinib resistance, and structural modeling work predicted that it would cause steric hindrance of gefitinib binding.

Together with results from patients with gastrointestinal stromal tumors and chronic myeloid leukemia, these findings show that somatic mutations in EGFR are likely to be responsible for relapses in patients who are at first responsive to gefitinib or erlotinib. Kobayashi et al. advise, therefore, that it might be appropriate to incorporate repeated biopsies into clinical studies in this area. They also note that results from a biochemical screen of alternative EGFR inhibitors identified a compound $(C L-387,785)$ that appeared to overcome resistance rendered by the T790M mutation. The characterization of resistance mutations might, therefore, pave the way for the development of effective second-generation EGFR inhibitors.

Original article Kobayashi S et al. (2005) EGFR mutation and resistance of non-small-cell lung cancer to gefitinib. New Engl J Med 352: 786-792

\section{Long-term follow-up of a smoking cessation intervention}

It is clear from cohort studies that stopping smoking has a positive effect on mortality risk, but there are few randomized data on this subject. The Lung Health Study compared mortality rates in smokers who were randomized to a smoking-cessation intervention program or to usual care (no intervention). Long-term results from the study have recently been published.

The trial included 5,887 volunteers from 10 clinical centers in Canada and the US, who smoked an average of 31 cigarettes per day. All had evidence of mild to moderate airway obstruction but were asymptomatic when the study began. Those randomized to the intervention group $(n=3,923)$ underwent a 10-week smoking-cessation program based on nicotine gum, group sessions and advice from the physician. Those in the usual-care group $(n=1,964)$ received no such interventions. Participants were followed up at annual visits for five years during the intervention phase of the trial, and then by telephone every 6 months, for up to a total of 14.5 years.

In an intention-to-treat analysis, all-cause mortality was significantly higher in the usualcare group than in the intervention group (hazard ratio $1.18,95 \% \mathrm{Cl} 1.02-1.37$ ). This was despite the modest success rate of the smoking-cessation program. (At five years, 21.7\% and $5.4 \%$ of participants had successfully given up smoking in the smoking intervention and usual-care groups, respectively.) The mortality benefit to the smoking intervention group was seen across several major categories of causes, including lung cancer, coronary heart disease, 